Agencies: Issues for consideration. Administration in Social Work, Vol. 30, № 4. Pág. 12

PEROLD, H. y TAPIA, M.N. (editoras). Servicio Cívico y Voluntariado en Latinoamérica y el Caribe Solidario, Buenos Aires; Johannesburg Volunteer and Service Enquiry Southern Africa; St. Louis, Washington University, The Center for Social Development Washington DC, Innovations in Civic Participation. Pág. V-VI. Extraído el 14 de marzo de 2011: http:// www.service-enquiry.org.za

QUIJANO, S.; CORNEJO, J.M.; YEPES, M. \& FLORES, R. (2005). La calidad de los procesos y recursos humano (CPRH) como componente de la calidad del sistem humano de la organizacion. conceptualizacion y mediFacultad de Psicologí, Universidad de Bacelona 7 -

REDD, P. \& SELBEE, L. Distinguishing Characteristic of Active Volunteers in Canada. Nonprofit and Voluntary Sector Quarterly 2000; № 29. Extraído el 23 septiembre 2009: http://nvs.sagepub.com/cgi/conten abstract/29/4/571

RíOS, R. Capacidad organizacional y calidad de la Intervención Social. Perspectivas CEES-UC No 6. Documentos de trabajo. Centro de Estudios de Emprendimientos Solidarios, Pontificia Universidad Católica de Chile. Diciembre 2008, Santiago, Chile.

ROJAS, G. (2004). Fundación Gesta: Una aproximación Mad. No11 Septiembre 2004. Departamento de Antropología. Universidad de Chile. Extraido el 22 agosto 2011: www.revistamad.uchile.cl/11/paper07.pdf

SALAMON, L. et al. (2006). Estudio Comparativo del Sector Sin Fines de Lucro. Santiago, Chile. Abril.

SEPÚLVEDA, N \& MUÑOZ, A. Voluntariado y edades: Observaciones desde la juventud, la adultez y la vejez voluntaria de la ciudad de Santiago. Revista MAD, N 20, 2009. Universidad de Chile. Santiago, Chile.

TARAZONA, D. (2004). Acercamiento a la experiencia subjetiva del voluntariado social en organizaciones juveniles. Revista Flectrónica del Instituto Psicología Desarrollo, Página 2 de 12 Año I, Número 3, Diciembre. Disponible en: http://sicj.cepal.org/publicaciones/ ficha/:id=231 el 22 de Agosto 2011 .

THOMPSON, A \& TORO, L. (2000). "El voluntariado social en América Latina. Tendencias, influencias, espacios y lecciones aprendidas". Revista Sociedad Civil, Mexico. Documento incluido dentro de la Biblioteca Digital de la Iniciativa Interanticana de Capital soDesarrollo (BID).
VAUGHAN \& ARSNEAULT (2003). Nonprofit Federalism: Implications of the Partnership Between Governments and Not-for-Profit Organizations in the Association Annual Meeting. Chicago, Illinois.

VECINA, M.L. (2001). Factores psicosociales que influyen en la permanencia del voluntariado. Tesis doctoral no publicada, Universidad Complutense de Madrid.

VERNIS, A. Tensiones y retos en la gestión de las organizaciones no lucrativas. Revista Española del Tercer Sector. Editada por Fundación Luis Vives. N 1 1 Octubre - diciembre 2005. Extraido el 22 de junio 2011:

ZULUETA, S. (2003). La evolución del voluntariado en Chile entre los años 1990 y 2002. Tesis para optar al grado de Magíster en Sociología, Pontificia Universidad Catolica de Chile, Santiago, Chile.

\section{Las prácticas de participación política de dirigentes de Centros de Estudiantes de Trabajo Social en Universidades Privadas}

Practices of political participation within students representations organizations leaders'

Alejandra I. Santana L.

Directora de la Unidad de Mejoramiento de la Docencia, Universidad Autonoma de Chile. Trabajadora Social, Magister en Psicologia, Magister en Educación, Doctor @ en Ciencias de la Educación. Correos electrónicos: asantana@uc.cl / alejandra.santana@

Natalia Hernández M.

Academica del Departamento de Trabajo Social, Universidad Alberto Hurtado. Trabajadora Social, Magister en Trabajo Social, Estudiante de Doctorado en Trabajo Social. Correos electrónicos: nehernan@uc.cl / nhernand@uahurtado.d

\section{Resumen}

En un escenario sociocultural contemporáneo que ha transformado las diversas formas de participación del mundo juvenil, este articulo ofrece una aproximación teórica y empirica a las prácticas de participación politicas de dirigentes de Centros de Estudiantes de Trabajo Social en Universidades Privadas.

La investigación se orientó a conocer qué expresiones han ido conformando las prácticas políticas de estos jóvenes, indagando en: el significado de la imagen de dirigente, la participación y la práctica politica, las estrategias de participación, las apreciaciones referentes a los partidos politicos y la relación de los centros de estudiantes con sus respectivas federaciones en el contexto universitario. Para esto se trabajó mediante una metodologia cualitativa recogiendo los datos a través de focus group y analizándolos a través de matrices de contenidos temáticos.

Los principales hallazgos apuntan a reconocer que el rol de dirigente se relaciona con su identidad juvenil y por tanto con su habitus. Poseen una noción amplia de participación política relidadojua y pol ejercicio de la ciudadania la práctica política es diversa, buscando la participació en si misma Las estrategias usadas corresponden a acciones concretas y acotadas que buscaión participacín y representatividad de sus compañeros La relación con sus federaciones ouncan la paricipaín y repa

Palabras clave. Jóvenes, participación politica, universitarios.

Summary

In a socio cultural and political scenario that has changed the socio-political forms of participation of the youth world, this article offers a theoretical and empirical approximation to the practices of political participation of leaders of students' organizations in Private Universities in Chile.

The research focused on knowing what expressions have shaped the political practices of these young people, looking into. the meaning of the leader image, the participation and political practice, participation strategies, the significances concerning political parties and the relationship of students organizations with their students federations in the university context. For this purpose, we worked using a qualitative methodology, collecting data through focus groups and analyzing them using thematic content matrixes.

The main findings point to recognize that the role of leader is related to its youth identity and thus their habitus. They have a broad notion of participation policy, related to the exercise of citizenship. Political practice is diverse, and it's valued in itself. The strategies used correspond to concrete and delimited actions, which aim to promote participation and representation within other university students. Finally, the relationship with their federations or university support units can be stressful, evaluated by these leaders as mechanisms to control them.

Key words. Youth, political participation, university students. 


\section{Presentación}

Los procesos de modernización y globalización han significado grandes transformaciones sociales, culturales y económicas, afectando a la sociedad en sus múltiples esferas, influyendo en la conformación de un sujeto contemporáneo. En las sociedades latinoamericanas, uno de los sujetos que aparece vivenciando un estado de opacidad permanente corresponde a los "jóvenes". Esta opacidad hace referencia a la socialización que se posee desde la matriz correspondiente a las miradas adultas, las cuales suelen invisibilizar la elaboración del sujeto social juvenil ${ }^{1}$.

Uno de los ámbitos en que los jóvenes se han visto transformados, en su identidad y en las relacione que establecen con sus pares y con el mundo adulto, lo constituyen las posibilidades y el cariz de la participación social, y particularmente, la participación política.

Ahora bien, se habla de participación política en su globalidad, sería interesante ver cómo se está manifestando este accionar en los espacios más cotidianos de los jóvenes, como lo son los lugares donde se desarrollan parte de sus procesos de formación. Cobra interés particular, tomar como espacio central, los programas de pregrado de Trabajo Social, de universidades privadas ${ }^{2}$

Este artículo busca dar conocer qué expresiones han ido conformando las prácticas políticas de jóvenes dirigentes estudiantiles de la carrera de Trabajo Social en el contexto universitario privado, a través de las vivencias de los dirigentes de dos Centros de Estudiantes de Trabajo Social de la región metropolitana, respecto a su participación política. El texto desarrolla una aproximación teórica y empírica a la participación política de los jóvenes. Se estructura, inicialmente, presentando antecedentes relevantes que posibilitan la conformación del fenómeno social de interés: la participación política

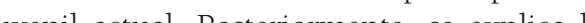
dología usada en el ejercicio empírico de captar las subjetividades de los jóvenes. Finalmente, se presentan los principales hallazgos en diálogo con el marco referencial ofrecido y las principales conclusiones.

\section{Antecedentes}

El contexto democrático nacional, podría hacer suponer que la participación social, en especial la de carácter político, sería mucho más activa y presente en la dinámica social. Sin embargo, "la política pareciera no seducir a los jóvenes, pero tampoco a los adultos, situación que se tematiza como una "crisis de la política", producto de una institucionalidad que se no adapta a las nuevas dinámicas de la sociedad chilena (crisis de representatividad, sistema electoral binominal, conductas políticas irregulares coliticas irregulares y/o deshonestas, etc.)" (Garretón, 2004 en Maturana, 2007, pág. 117)

Hoy, lo político para los jóvenes tiene sentido, en cuanto se refiera "al encuentro con otro, a la gestión de ese encuentro, a establecer las condiciones que permiten que este encuentro sea efectivamente igualitario y democrático, reconociendo la diferencia. Ponerse de acuerdo generand encturas incluyentes, que han dejado de lado la producción discursiva por poco práctica y se han centrado en la acción y la articulación como el método por excelencia para lograr un cambio" (Saintout, 2009, pág. 147).

De acuerdo al Informe de Juventud en Iberoamérica de la CEPAL (2007), las formas de participación juveniles, en general, han cambiado. Se sigue valorando la participación como un mecanismo de autorrealización y de obtención de logros. Se señala que la participación de los jóvenes tiende a darse en ámbitos locales, fuera de los partidos políticos, asumiendo pequeña escala, menos horizonte temporal y alcances más modestos de las pretensiones de cambio.

Particularmente, en lo referido a la participación política, Gamboa \& Pincheira (2009) señalan que los jóvenes están cambiando las formas tradicionales de experimentar la participación socio - política hacia "otras" formas que empiezan a proliferar. Un ejemplo de ello lo conforma la noción de "ciudadanía activa", transversal y descentrada, construida y ejercida en colectivo, y a partir de la multiplicidad de experiencias territoriales y situaciones cotidianas que identifican la subjetividad juvenil contemporánea. Este tipo de democracia surge desde la "necesi-

Las juventudes en si mismas, han sido invisibilizadas en su complejidad, puesto que desde las ciencias no se ha logrado develar los infinitos componentes que hacen de ellas más que una categoría proveniente de las lideres de corte positivista. La palabra juventud, cambios biologicos, psicológicos y sociales de un ser humano. Además, esta concepción ha estado ligada a un tiempo etáreo especifico que brinda märgenes, en función de los contextos sociohistóricos que se analicen. En Duarte. K: juventud o Juventudes? Versiones, trampas, pistas y ejes para acercarnos progresivamente a los mundos juveniles; en Revista Pasos n n' 93 , San José, Costa Rica.1999.

Para la presente investigación, es relevante observar los ejes de lo politico, la participación y asociación que se desarrollan desde la disciplina de Trabajo Social, en sus diversos programas de formación; puesto que, se reconoce que dichos ejes son elementos constituyentes de discusión del aspecto disciplinar que se ha reconoce en la actualidad.

dad de impulsar entre las nuevas generaciones una conciencia de la responsabilidad que tienen en la marcha de los asuntos de la comunidad a la que pertenecen, superando el escepticismo predominante entre los jóvenes que les lleva a considerar las cuestiones de índole política como algo lejano y extraño a sus intereses. Para conseguir impulsar entre los jóvenes esta concepción más activa de la vida cívica se confía básicamente en los procesos de aprendizaje tanto formal como informal, que les permitan adquirir las capacidades y competencias necesarias para poder participar eficazmente en una sociedad cada vez más compleja y plural" (Benedicto y Moŕn 2002, pág.20). De acuerdo acuerdo a las cifras aportadas por Quinta Encuesta Nacional de la Juventud (2008), la orientación política de los jóvenes chilenos se manifiesta en un $36,9 \%$ que no adscribe a ninguna, un $19,8 \%$ que se orienta a la izquierda y un $11,8 \%$ a la derecha.

En cuanto a los ámbitos específicos de interés por participar, en relación a organizaciones sociales, los jóvenes se inclinan principalmente por voluntariado, deporte, religión, defensa de ideales y movimientos culturales (INJUV, 2008). De la totalidad de jóvenes chilenos, un 1,7\% participa en política, y solo un $4 \%$ de este total participa en Centros de Alumnos (CAA) ${ }^{3}$. De ellos, un $57 \%$ considera que la democracia es preferible a cualquier otra forma de gobierno.

Ahora bien, en lo relativo al contexto universitario, cabe destacar que la "universidad" es una de las instituciones de mayor confianza para los jóvenes, el $62 \%$ de ellos lo declara; en esta misma línea los profesores universitarios constituyen uno de los actores institucionales en los cuales los jóvenes más confían, un 56\% de ellos, lo avala (INJUV, 2008).

Si bien se cuenta con estas cifras, sería necesario un abordaje que permita profundizar en la comprensión de la vivencia de participación política en el contexto universitario en una de las unidades más acotadas de organización como son los Centros de Estudiantes (CCEE), a fin de ir develando las nuevas vivencias, la forma en que se van expresando y los sentidos que tienen para los jóvenes.

En palabras de Baeza (2006) "una educación de calidad exige reconocer a actores y autores implicados. Exige, en el caso de los estudiantes, escuchar su voz, que se articula desde sentimientos y vivencias, que no necesariamente se expresa en los códigos acostumbrados del mundo adulto. Es un lenguaje propio que para escucharlo previamente hay que reconocerlo (...) su valor no radica en la potencialidad de ser ciudadanos del futuro; son ciudadanos y ciudadanas ahora" (pág. 295).

\section{Participación política juvenil en espacios universitarios organizados desde los aportes de Bourdieu}

En consideración a lo expuesto, es posible acercarse a las significaciones que las y los jóvenes brindan a las participación política en los Centros de Estudiantes de la carrera de Trabajo Social de la universidades privadas a través de las claves de Bourdieu, particularmente desde las nociones de campo y habitus. Ambas aportan la riqueza de considerar lo social y lo subjetivo en la comprensión de los fenómenos sociales. Junto a ello, se enfatizarán aquellos antecedentes que permitan situarse en la actual relación jóvenes/política, y las actuales prácticas asociativas y de representación desarrolladas por los jóvenes en Chile.

Como se mencionó, la aproximación a la participación política de las y los jóvenes estudiantes, se utilizará la perspectiva sociológica de Bourdieu. Esta mirada ofrece la riqueza de ver dos formas de existencia de lo social: "las estructuras sociales objetivas construidas en dinámicas históricas -los campos- y las estructuras sociales interiorizadas, incorporadas por los individuos en forma de esquemas se percepción, valoración, pensamiento y acción -los habitus." (Rizo, 2006, pág. 1 ).

\section{La Universidad como campo}

En relación al campo, Bourdieu lo concibe como "microcosmos que poseen desafíos, objetivos e intereses específicos" (Chauviré \& Fontaine, 2008). En este caso, se hablará de la universidad como el campo en el que se da el juego de ser estudiantes, particularmente, de ser dirigentes estudiantiles. Ese contexto tiene su propia dinámica y lógica interna y dependiendo del sello que deseen impregnar en sus estudiantes, se planteará con más o menos matices el desafío de formar profesionales de excelencia y aportar de determinada manera a la sociedad. Ahora bien, se ha acotado la investigación a las universidades privadas, lo que suma un nuevo ámbito de diferenciación. Muchas de ellas no solo buscan 1 excelencia académica sino que poseen fines de lucro y funcionan más como empresas que como espacios para el saber y el conocimiento universal.

A los campos desde su autonomía les concierne a la vez, el ser y el conocer, "al diferenciarse, el mundo 
social produce la diferenciación de los modos de conocimiento del mundo; a cada uno de los campos corresponde un punto de vista fundamental sobre el mundo que crea su objeto propio y que encuentra en sí mismo el principio de comprensión y de explicación conveniente a dicho objeto" (Op cit, 2008, pág 14) Se "s da forma en lampo de la universidad privada, de un forma que ahr tiene sentido; pero tarien, desuna forma que ahí tiene sentido; pero también, desde esa posición y en esa ubicación, se ve y explica el mundo, el mundo relevante a ese campo. Desde ahí los dirigentes estudiantiles se constituyen como tales, se mueven en torno a las autoridades universitarias, a sus profesores y st pares; desde ah "piensan" las necesidades de los estudiantes, el tipo de actividades que realizan, entre otros aspectos.

"Los campos se presentan como 'espacios estructurados de posiciones', las de los agentes que trabajan en esos campos de fuerzas, "cuyas propiedades dependen de su posición en esos espacios y que pueden ser analizadas independientemente de las características de sus ocupantes" (Op cit, 2008, pág. 15). La estructura de campo está determinada por estas posiciones, es decir, estarán los estudiantes como dirigentes estarán las autoridades universitarias, ubicados en posiciones opuestas generando campos de fuerzas, luchas por el poder esperables en ese campo.

Los agentes son parte de un campo y se inscriben en él a partir de ciertas disposiciones de una manera de ser requerida por la dinámica propia del campo; "la lógica específica de un campo se instituye en el estado incorporado en la forma de habitus, más precisamente, de un sentido del juego; "cada campo se caracteriza por la prosecución de un fin específico, apto para favorecer investiduras igualmente absolutas en todos aquellos que poseen las disposiciones recueridu" (Op cit, 2008, pág. 16). De esta manera, en el campo de la universidad, quienes desempeñen un habitus acorde a las disposiciones de ese campo lograrán el fin de "titularse profesionalmente". Esa será su investidura, ese sera el capital específico por el cual se ha jugado. Por otra parte, desde las autoridades universitarias se habrá aportado con profesionales de excelencia al país, y, de paso, se habrán incrementado sus arcas con el pago de aranceles por cinco o más años.

"La lógica de un campo, la jerarquización de los intereses y la discriminación de los objetivos pertinentes instituidos, funcionan así como el trasfondo impensado de las prácticas de los agentes: form lo implicito que está en el fundamento mismo de todas sus acciones, colaboraciones y, paradójicamente, de todos los conflictos y desacuerdos" (Op cit, 2008, pág. 18). Así, los dirigentes estudiantiles universitarios jugarán en ese micro espacio social, sobre los acuerdos implícitos compartidos, con las disposiciones necesarias (generadas incluso en el campo) para jugar allí Ahora bien, esas disposicioa expresan en la idea de habitus. De esta manera, la noción de campo facilita la comprensión del contexto complejo -y a la vez diferenciado- donde emergen las nuevas prácticas de participación política de los dirigentes estudiantiles, estructuras y estructurantes de ellas.

\section{El Habitus del Dirigente Estudiantil}

La noción de habitus apela a "un sistema de disposiciones duraderas, que funcionan como esquemas de clasificación para orientar las valoraciones, percepciones y acciones de los sujetos. Constituye también un conjunto de estructuras tanto estructuradas como estructurantes: lo primero, porque implica el proceso mediante el cual los sujetos interiorizan lo social; lo segundo, porque funciona como principio , pág. 1). El estudiante universitario que posee el rol de dirigente cuenta con un habitus específico, que responde a las disposiciones condicionadas por el campo de la universidad. Tiene cierto grado de estabilidad, en tanto responde a un período de tiempo, mientras dura el mandato de la federación de estudiantes o del CEE -como el caso en estudio-. Desde ahí se diferencia del resestinguido en el contexto universitario, piensa de cierta manera, actúa de una manera especial, también conforme a su habitus, sus prácticas lo expresan. Que ellos llamen a asambleas, organicen actividades culturales, representen a sus compañeros dentro y fuera del contexto universitario es constituyente del habitus. En palabras de Bourdieu (1988), "la identidad social se define y se afirma en la diferencia" (pág.170).

El habitus, "en tanto que disposición general y transportable, realiza una aplicación sistemática universal, extendida más allá de los límites de lo que ha sido directamente adquirido, de la necesidad inherente a las condiciones de aprendizaje: es lo que hace que el conjunto de las prácticas de un agente sean a la vez sistemáticas, porque son producto de la aplicación de idénticos esquemas, y sistemáticamente distintas de las prácticas constitutivas de otro estilo de vida" (Bourdieu, 1988, pág. 170). En relación a esta descripción, sería interesante detenerse en que el habitus puede conformarse por una disposición general y que a la vez es transportable; por ejemplo, algunos dirigentes estudiantiles universitarios que en este momento están ejerciendo algún cargo en los Centros de Estudiantes traen un habitus dirigencial desde sus épocas de colegio, donde algunos en la educación básica y media fueron presidentes de cursos y/o en la educación media fueron actores protagónicos en el movimiento estudiantil pingüino en Chile durante el año $2006^{4}$. Transportan su habitus y su práctica al campo de la universidad, donde es aplicado sistemáticamente en el campo de juego, estructurándose conforme las particularidades de este campo, que posee desafíos, objetivos y fines diferenciados a los que tenía el campo de la escuela o liceo. El habitus "es un conocimiento incorporado, hecho cuerpo, adherido a los esquemas mentales más profundos, a los dispositivos de la pre-reflexión, del "incosciente social", con los que las personas guían la mayor parte de sus prácticas sin necesidad de racionalizarlas, pero adecuadas a un fin racional" (Rizo, 2006, pág. 2). El habitus de dirigente estudiantil trae consigo un conocimiento, una perspectiva para ver el mundo, los dirigentes -en su mayoría- acuñan un pensamiento crítico acerca de la sociedad, los políticos, aspectos del funcionamiento de sus universidades y unidades académicas a las que pertenecen; al profundizar más en ellos, se podría identificar formas de hablar, de vestir, de posicionarse socialmente. Si hay una situación que demande el actuar de los dirigentes en la universidad, de ellos se espera que tengan una opinión, que informen a sus compañeros e incluso que los guien en el curso de ciertas acciones, que los representen; esto se relaciona estrechamente con la multidimensionalidad del habitus.

Para Rizo (2006), "el habitus tiene un carácter multidimensional: es a la vez eidos (sistemas de esquemas lógicos o estructura nes morales), hexis (registros de y aisthesis (gustos, disposición estética). Portos) el concepto engloba conjuntamente los planos cognitivos, axiológicos y prácticos y cuestiona las distinciones dicotómicas tradicionales entre las categorías lógicas y éticas, por una parte, y el cuerpo y el intelecto, por otra" (pág. 2). De esta manera, se pueden tener intuiciones en relación a cómo se expresa el habitus del dirigente estudiantil de Trabajo Social en universidades privadas. Sin embargo no se ha explorado suficiente en estas multidimensiones, ¿de qué manera se concibe el dirigente estudiantil en el contexto universitario? icómo ve el mundo social? icómo se relaciona desde su habitus con el resto de la comunidad educativa con la cual comparte un campo? ¿qué valores están a la base de su acción? ¿cuál es su fin en tanto dirigente?

El habitus, siendo producto de la historia, es un sistema abierto a disposiciones que se confrontan permanentemente por ellas, de forma duradera pero no inmutable (Op cit, 2006, pág. 2), con tendencia la actualización. En relación a la característica dinámica y actualizable del habitus, cobra relevancia la noción de "trayectorias de vida" de los jóvenes, lo que ayuda a comprender que su habitus actual se llega a conformar como tal a partir de sus historias familiares y de personas y sucesos significativos que -incluso en otros campos- generan y fortalecen las disposiciones actuales a jugar en el campo de la universidad, el habitus de dirigente de Centro de Estudiantes.

Luego de haber realizado esta aproximación a la vivencia del dirigente estudiantil de Trabajo Socia desde la mirada de Bourdieu, no queda más que abrir preguntas en su clave; ¿Qué características posee este habitus? ¿cuáles son las nuevas prácticas sociales que lo expresan? ¿qué relación tienen con un orden social mayor que lo contiene y determiha estructurantemente? ¿qué vínculo se encuentra entre el habitus y la identidad de los jóvenes dirigentes? ¿qué particularidades posee el campo de la universidad en la emergencia de este habitus de dirigentes estudiantil?

\section{Participación política juven}

en clave contemporánea

La ciudadanía activa entre los jóvenes

La perspectiva de ciudadanía activa surge como consecuencia tras la época de optimismo de post-

\section{Algunas citas que evidencian esta ided.}

(...) mi mamá siempre ha tenido ingerencia en lo judicial, no tanto social, sino a nivel de iglesia y por eso siempre tuve como una

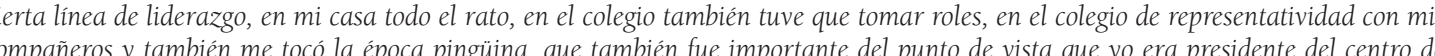
estudiantes del colegio y tuve que hacerme cargo de muchas cosas, de entrevistas, de hacer lineas de acción y yo creo que todo nace desde mi infancia, de mi familia, de cómo se gestó este liderazgo y cómo también la gente ha esperado de mi que los represente" (hombre 3). "Mi mamá participó en un sindicato en su trabajo durante muchos años, por to tanto las conversaciones de almuerzo eran siempre generar pensamiento crítico... recibir muchos estimulos externos, en mi familia hay gente que participa en politica y siempre las conversaciones desde chica yo recuerdo que eran enfocadas a que esto no puede ser, que los derechos, los deberes, que los derechos, que los deberes, también siempre mi entorno fue asi (...) (mujer 1 ). 
guerra en Europa, cuya máxima expresión había sido el desarrollo del Estados de los Bienestar y la construccion de una ciudadania basada en el reconocimiento de los derechos sociales. Así, cuando el proyecto socialdemócrata de los años ' 50 y '60 da sintomas de de referencias intelectuales y preocupaciones colectivas, que intentaron de comprender estos nuevo escenarios, destacando la necesidad de constru una ciudadanía activa (Benedicto y Morán, 2002).

La vida cívica de las democracias avanzadas exige, de manera cada vez más evidente, una nueva configuración en la que el ciududuno esté más presente, desarol interacciones con las instituciones. Ahora bien, esterciones con las instituciones. Ahora bien, este contexto, llevado a la vivencia de los jóvenes, tiende a replicar la matriz adultocéntrica, apelando a un ciudadano activo que es adulto o que se prepara para serlo. De esta manera, un mirada alternativa apostaría por definir la juventud como un proceso de producción de ciudadanos (Morán y Benedicto, 2000).

La ciudadanía activa concede una especial relevancia a la dimensión política, contrariamente a lo que ocurría en otros momentos históricos en los que el principal reto consistía en desarrollar el componente social de la ciudadanía de los jóvenes. Esta significa básicamente presencia y probgonismo de jóvenes, desde los jovenes, desde su condición de ciudadanos, en el desarrollo de los procesos sociales y políticos de la sociedad en la que viven. Dos son, por tanto, los elementos fundamentales a tener en cuenta para hablar de ciudadanía activa: presencia y protagonismo.

\section{Los jóvenes y la política: nuevas formas} de articulación

La participación política ha tenido vuelcos respecto a lo que fue en décadas anteriores. En relación a la vinculación de los jóvenes con la política, se dice que "(...) los jóvenes aparecen a los ojos de la opinión pública como un colectivo poco interesado en este tipo de asuntos y, lo que es más importante, muy poco proclive a asumir responsabilidades en la esfera pública" (Benedicto, 2005 pág. 1).

Claro está que lo que les sucede a ellos no está aislado de lo que les sucede a otras generaciones. Es así como una primera condición con respecto a la po- lítica y los jóvenes que debe señalar es su "malestar profundo con las instituciones tradicionales de la política y sus representantes en un tiempo histórico donde amplios sectores sociales lo ponen en duda" (Saintout, 2009, pág. 105)

En el cuestionamiento a las formas tradicionales de ejercicio de la política "es la tendencia a negarse a ser representados por partidos políticos, éstos son funcionales a los "políticos", caratulándolos comúnmente como corruptos o mafiosos (...) La crítica a la política aquí no es ideológica, sino ética; no plantea una alternativa ni técnica ni partidaria a no plantea una alernativa ni tecnica ni part (Op cit, 2009, pág. 108)

Para Rossana Reguillo (2000) la relación entre juventud y política se destaca por el declive de las formas políticas decimonónicas que han predominado en occidente, más un cierto deterioro de la ciudadanía, concibiendo esta formateada por los poderes oficiales, que abarcan una dimensión política (derecho a sufragio), una dimensión social (ligada al Estado social - benefactor) y una dimensión civil (libertades individuales), invisibilizando la trama compleja y diversa de las diferentes manifestaciones intersubjetivas imbricadas en torno al tejido cultural (jóvenes, niños, mujeres, sexualidades diversas). (Reguillo, 2000 en Gamboa \& Pincheira, 2009, pág. 34).

Las transformaciones en las prácticas políticas juveniles responden -entre otros elementos- a la diversidad contenida en las culturas juveniles, donde desde sus diferentes estilos van permeando a nuevas formas de vivir "lo político".

Las "nuevas formas de implicación cívica de los jóvenes se caracterizan por su carácter fluido y poco diferenciado, por la mezcla de canales de expresión, por la pluralidad de espacios y significados que les llevan a considerar desde la participación política más o menos formalizada a la participación social organizada o a diversas formas de voluntariado" (Benedicto, 2005 pág. 9). Así, por ejemplo, en relación a colectivos juveniles se destaca "la capacidad que han desarrollado muchos de ellos de generar organizaciones autogestionadas, horizontales, donde son responsables de sí mimos sin tener por encima ningún tipo de autoridad vertical" (Saintout, 2009 pág. 109)

Estas tendencias emergentes de participación juvenil, que se expresan en estas nuevas formas de

Cuando se hace referencia a la noción de participación politica juvenil, es interesante mirar los planteamientos de Garretón (2001), quien indica que la politica ya no es el único referente (como lo fue en décadas pasadas), pues actualmente se encuentran diversos aspectos que posibilitan comprender los elementos identitarios y de movilización. No se puede invisibilizar que las y los jóvenes han visto fragmentada y mermada esta forma de participación. Según el mismo autor, tiene una estrecha relación con la desilusión de una democracia no representativa. mplicación cívica, suponen la participación de "sujetos autónomos que experimentan diferentes maneras de influir en los procesos sociales y políticos, contrariamente al modelo clásico de los movimientos juveniles que utilizan la acción política como instrumento para la emancipación" (Benedicto, 2005, pág. 10).

Así, estas nuevas formas de participación responden a formas de concebir la ciudadanía. "Hoy cobra mayor preponderancia una "ciudadanía activa" transversal y descentrada, constituida y ejercida en colectivo, y a partir de la multiplicidad de experiencias territoriales y situaciones cotidianas que identifican a la subjetividad juvenil contemporánea" (Gamboa \& Pincheira, 2009, pág. 35).

Desde esta ciudadanía activa, se exploran espacios no tradicionales de expresión política, relevando la dimensión de la subjetividad en el espacio público. "En unas sociedades donde se replantean los pactos preexistentes y se aceleran los procesos de individuación, los jóvenes no solo niegan la política concebida tal cual lo hicieron sus padres, sino que la reemplazan por una puesta en escena de aquello que antes estaba "guardado": la propia subjetividad" (Saintout, 2009, pág. 107).

En los sentidos que mueven a los jóvenes, estos responden a demandas mucho más acotadas e individualizadas; ellos ven la efectividad de sus acciones en ámbitos que están a su alcance, donde pueden reconocer las transformaciones y dónde ellos mismos son los principalmente transformados. "No es que no conozcan los "grandes problemas" de toda la sociedad (...), pero cuando piensan que es posible transformar esta realidad, la vía es una vía personal transformarse uno mismo para transfornarel mundo la transformación interior ( . . . Siguen creyendo en la solidaridad, en la preocupación por el otro, pero esta solidaridad hoy pareciera tener límites más cercanos, o más concretos: es la solidaridad con los amigos, con los miembros del grupo, con la propia familia (...). Hoy la solidaridad la definen casi "cara a cara", es decir, a partir de la identificación de la identidad personal de aquellos con los cuales se es solidario" (Op cit, 2009, pág. 107).

Respecto al uso del poder, este es más horizontal y flexible; los dirigentes estudiantiles señalan la horizontalidad como un valor, la polifuncionalidad de sus cargos, y la coordinación por sobre un control jerárquico. En la mayoría de las nuevas asociaciones comunitarias y que comparten muchos grupos juveniles (...) se da un poder que circula, que no se ejerce de arriba hacia abajo y que es redefinido a partir de su no exterioridad con respecto a los su- jetos; no como algo de lo cual hay que apoderarse sino como una dimensión que hace el tejido social (Op cit, 2009, pág. 110)

En cuanto a la temporalidad, los jóvenes la viven de una forma distinta a las generaciones pasadas: (...) La factibilidad de generar proyectos alternativos se choca con un tiempo del absoluto presente donde el futuro ya llegó, no deja de llegar nunca y ahora todo es ahora (...). Las acciones se reducen al momento, porque luego no se sabe qué podrá pasar (Op cit, 2009, pág. 110).

Ahora bien, se ha revisado la relación entre jóvenes y política desde el escenario contemporáneo iberoamericano. Sería relevante profundizar en las estrategias organizativas de las agrupaciones juveniles chilenas.

\section{Estrategias de organización de agrupaciones} juveniles

De acuerdo al trabajo de Andrea Gamboa e Iván Pincheira (2009) respecto a organizaciones juveniles, se logran dilucidar algunas características asociadas las prácticas de asociación y de organizaciones de estas agrupaciones. Si bien no hace mención directa a los CCEE u organizaciones estudiantiles, concuerd con múltiples dimensiones confrontadas en el diálogo con los dirigentes estudiantiles de Trabajo Social

En cuanto a las estrategias para la organización, se reconocen:

- La definición y lugares de la politicidad. Implica el desarrollo de reflexiones acerca de las formas de acción que les serán propias, lo que quieren y no quieren, sus objetivos y como cumplirlos.

- La (in) visibilidad. Se puede considerar una línea de acción, la visibilidad de las agrupaciones y sus acciones; implican plantearse objetivos, manejo de recursos diferenciados y esfuerzos. Se expresa en el manejo de la imagen de sí como organización, qué se quiere mostrar de ella. Para esto muchas veces se usan los recursos virtuales como Fotolog, Facebook, blog, foros, entre otros.

Ahora bien, la distribución de roles y funciones en virtud de su participación y de cómo éstos son asignados se caracteriza por relaciones marcadas por el respeto, lo que se visualiza en las características y los espacios de las tomas de decisiones, así como en las críticas que nacen en el grupo a su propia manera de funcionar.

En relación a sus momentos y tiempos de coordinación, las agrupaciones tienen frecuencia de reuniones semanales, que puede cambiar conforme a alguna emergencia o requerimientos de una tarea 
importante. En los encuentros se trabajan aspectos de la vida colectiva, se dan reflexiones, definiciones del grupo, se tratan aspectos operativos del trabajo y se generan y mantienen lazos afectivos. Las reuniones pueden ser presenciales o virtuales y pueden tener distint las reuniones para coordinarse, las reuniones que implican organizar asambleas con los estudiantes de toda la carrera o el acordar encontrarse en messenger a determinada hora, especialmente en la noche, para tomar alguna decisión operativa.

Respecto a las estructuras, la mayoría de las agrupaciones se declaran horizontales, más bien como una crítica a la verticalidad. Para establecer defini áreas de trabajo permanente sin establecer líneas de mando verticales muchos grupos designan "coordinaciones": hay una general, y coordinaciones dedicadas a distintos temas y responsabilidades. Po ejemplo, en uno de los CCEE, participantes de este estudio, se explicitaba el uso de coordinaciones para mantener una era, considerando que esta contaba con 500 estudiantes distribuidos en jornadas diurna y vespertina. No obstante la autonomí y horizontalidad indicadas, no se puede dejar de reconocer lo indicado por Aranda (2000 en Baeza, 2006) respecto a que los movimientos estudiantiles se componen en su mayoría por una masa mayoritaria de jóvenes que participan esporádicamente

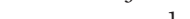
un grupo de activistas que continuamente estan levando a cabo acciones diversas que, de algu manera, mantienen la actividad del movimiento. La toma de decisiones se define por excelencia en asambleas, ya sea como estilo de conducción de la conversación grupal, o bien como asamblea propiamente tal. Sin embargo, también son críticos ante las dificultades de cómo concebir la asamblea cómo innovar en ella o cómo potenciar en ellas espacios más activos de participación.

En cuanto a la organización de actividades, impera la amistad a la hora de organizar, los cargos adquieren más relevancia y la función de cada quien es importante; ya que si fallan, arriesgan el éxito de actividad. La organización en red, por ejemplo, implica que cada quien se hace responsable por algo a lo mejor pequeño, pero muy importante para los fines de la acción. Es así como el compromiso y la responsabilidad son base.
Las nuevas formas de la organización estudiantil para hacer valer su opinión

Luego de haber revisado algunas características de las prácticas organizativas de las agrupaciones juveniles, se podrían acercar a las organizaciones centradas en las prácticas políticas de los dirigentes estudiantiles universitarios. Un aporte significativo, tiene -especialmente- para la organización estudiantil contemporánea, que hace tres años atrás fue un agente activo en la "revolución pingüina" en Chile el hecho de validar la instancia de "asamblea" como medio más eficiente de movilización, poniencomo medio mas efciente de move do en jaque el rol de "dirigente" versus el rol de "vocero" (Baeza, 2006).

Las organizaciones generadas o que asumen la lógica de la democracia directa poseen una estructura donde la participación de todos los integrantes de la organización es facilitada por su horizontalidad, la relevancia de la base, la consideración de espacios micro y la existencia de mecanismos de control para el aseguramiento del respeto a las decisiones tomadas.

Algunas características comunes de las organizaciones de democracia directa, incluidas en estas las organizaciones estudiantiles, son (Baeza, 2006):

- La inversión de la lógica piramidal tradicional, donde el vértice superior constituye el punto mayor de energía. En las organizaciones de estructura tradicional, es en la cúspide donde descansa habitualmente la tarea de proponer. motivar, conducir e incluso evaluar, situaciones todas que justifican el calificativo "dirigente", para quienes dirigen. En las organizaciones con democracia directa, los dirigentes son reemplazados por "voceros", que dan a conocer la voz de sus representados. Su tarea no es dirigir, sino ser portador de la voz de la asamblea.

- A diferencia también de los dirigentes, los voceros son "voceros revocables", es decir sobre ello está siempre la posibilidad de un rápido cambio Ello, a diferencia de la tarea dirigencial, donde el dirigente es elegido por un período por lo general no menor de tiempo, y donde el revocar su mandato no es un trámite fácil.

- La posibilidad de un alto y rápido control sobre los voceros ayuda, además, a una fuerte disciplina de respeto de los acuerdos.
- Los voceros, como uno entre sus pares, cumplen una tarea más dentro de la organización, la que, en su interior, articula un complejo tejido que posibilita a sus integrantes participar en numerosas áreas y comisiones de trabajo. Situación que aporta al involucramiento e identificación con la organización.

- La valoración de la igualdad de género al interior de las organizaciones, cuidado por la presencia femenina en las tareas de representación. Se cuida que tanto en las labores de vocerí con distribución de tareas diversas, exista equilibrio en términos de género.

Quienes revindican la democracia directa se oponen a la democracia representativa, cuestionándole que se basa en la elección de "representantes" que, una vez elegidos, se arrogan el derecho de "representar" sin ningun mecan de estos "dirigentes" por parte de las bases que los eligieron.

Las organizaciones estudiantiles, que asumen la lógica de una democracia directa, "por lo general, no se inscriben ni adhieren a un partido político en particular, sino que mantienen una autonomía en la ocing par acorde a su discurso de respeto a las diferencias e igualdad de las personas. Ello no implica que quienes integran estas organizaciones no posean posiciones político-partidistas, sino que sus posiciones son un elemento más de diálogo interno, en la democracia de la organización" (Baeza, 2006, pág. 290).

Para cerrar este apartado, aclaramos que es un marco referencial preliminar centrado en antecedentes más que en constructores teóricos que den un soporte en esa línea. Se buscó entregar un panorama

TABLA $N^{\circ} 1$

\begin{tabular}{|c|c|c|}
\hline UNIVERSIDAD & PARTICIPANTES & CARACTERÍSTICAS \\
\hline \multirow{3}{*}{ Universidad 1} & Hombre 1 , Presidente CEE TS & $\begin{array}{l}22 \text { años } \\
3^{\text {er }} \text { año de la carrera / Jornada diurna }\end{array}$ \\
\hline & Mujer 1, Vicepresidenta CEE TS & $\begin{array}{l}31 \text { años } \\
4^{\circ} \text { año de la carrera / Jornada vespertina }\end{array}$ \\
\hline & Hombre 2, Coordinador General CEE TS & $\begin{array}{l}21 \text { años } \\
3^{\text {er }} \text { año de la carrera / Jornada diurna }\end{array}$ \\
\hline \multirow{3}{*}{ Universidad 2} & Hombre 3, presidente CcEE & $\begin{array}{l}21 \text { años } \\
4^{\circ} \text { año de la carrera / Jornada diurna }\end{array}$ \\
\hline & Hombre 4, Vicepresidente CcEE & $\begin{array}{l}20 \text { años } \\
3^{\text {er }} \text { año de la carrera / Jornada diurna }\end{array}$ \\
\hline & Mujer 2, Tesorera CcEE & $\begin{array}{l}21 \text { años } \\
4^{\circ} \text { año de la carrera / Jornada diurna }\end{array}$ \\
\hline
\end{tabular}

general de la relación jóvenes - política en Iberoamérica, revisar algunas prácticas de organización y dinámicas propias de agrupaciones juveniles chilenas, para finalizar con un elemento característico movimiento estudiantil secundario, donde se valora la organización basada en la democracia directa. Estos referentes son valiosos a la hora de buscar explorar las prácticas políticas de los dirigentes estudiantiles universitarios, mostrando rutas a seguir en la comprensión de ellas.

\section{Acercamientos a las voces \\ de los protagonistas}

Para realizar este ejercicio de investigación se optó por una metodología cualitativa, que permitiera recoger los propios discursos de los estudiantes, y desde ellos, captar las prácticas políticas actuales y los significados atribuidos a ellas desde su perspectiva.

\section{Participantes}

Se invitó a participar de esta instancia a cuatro CCEE de universidades privadas de la Región Metropolitana; de esta convocatoria, dos aceptaron participar manifestando su voluntad vía correo electrónico, nvolucrándose activamente en el proceso de recolección de datos y validación de la información. Específicamente participaron tres integrantes de cada Centro, los que cumplieron con criterios de selección: Poseer un cargo al interior del CEE, cursar como mínimo el $6^{\circ}$ semestre de la carrera, tene experiencia de a lo menos un año como dirigente $y$ ener la voluntad de participar. A continuación se de los informantes: de los cambios organizativos que emergió con el 


\section{nstrumento}

Se trabajó en base a un Focus Group, el cual fue conducido mediante un guión de preguntas elaboradas de acuerdo a l cuales se basó en temáticas revisadas en la literatura respecto a participación política de jóvenes.

El Focus Group fue desarrollado en dependencias de la Universidad $\mathrm{N}^{\circ} 1$, a cargo de la investigadora más la ayuda de un observador externo, lo que facilitó el registro y la transcripción final.

\section{Análisis de los datos}

Para efectos del análisis se desarrolló un análisis de contenido temático donde se revisó frase a frase, permitiendo la emergencia de las dimensiones. Las dimensiones operaron como categorías que facilitaron la denominación de los contenidos y su clasificación.

De esta forma, se organizó por tópico y/o dimensión, tablas que dan cuenta de las categorizaciones principales, algunas de las cuales fueron explicadas complementándose con las frases textuales de los jóvenes. Los principales tópicos fueron: imagen del dirigente estudiantil, participación y práctica política del dirigente estudiantil, valoración de vocerías y asambleas, partidos políticos y "lo político" y relación CEE y Federación de Estudiantes Universitaria.

\section{Principales hallazgos}

El trabajo realizado con las y los jóvenes participantes posibilitó acercarse a las formas en que significan la participación política que ellos ejercen en los espacios organizados al interior de la universidad. Para la concreción del ejercicio se realizó un análisi descriptivo de los tópicos que orientaron el ejercici investigativo, siendo cuatro los ejes fundamentales: a) Aspectos asociados al significado de la imagen de Dirigente Estudiantil Universitario.

Respecto a la percepción que los propios dirigentes estudiantiles universitarios tienen de su imagen de "dirigentes", se logró apreciar dos dimensiones: una enfocada a la imagen que ellos mismos percibían y otra, enfocada en cómo creían que los otro los veían.

En relación a la imagen autopercibida, se hace mención a las posibilidades del rol, es decir, a la forma en que les permite canalizar sus intereses, les genera satisfacción personal e implica responsabilizarse de procesos. Por otra parte, se señalan las acciones mismas, plasmadas en roles: mediador, representante, líder, entre otros.
Desde la forma en que son vistos por otros, los dirigentes aluden principalmente a la forma en que -ellos perciben- son vistos por sus compañeros. Desde ahí, reconocen que un grupo los ve desde el rol de representarlos, de ser voceros y contar con in embargo, también reconocen que por algunos, son vistos como quienes no hacen nada o los asocian de manera prejuiciosa a ser 'comunistas'

Así explican la visión que hace referencia al rol que desempeñan: "(..) siempre me han visto como alguien que tengo que representar, (. ) lo más eficaz posible, que lengo que repente (...) lo mas efcaz posible, posible, pero en ge posible, pero en general yo considero que me ven como, como alguien que pueda tomar su voz y de trasmitirlo, y que no existan represalias para ellos, sin miedo". (Hombre 3).

Por su parte, desde la mirada contraria, se señala: "(...) yo creo que la gente nos sigue viendo como "esos cabros que están ahí como que a lo mejor no han hecho nada, esos cabros que de repente andan puro leseando, son cabros que no se preocupan de estudiar y andan haciendo otras cuestiones" (...)". (Hombre 2).

Y complementan: "(...) la gente cree por lo general que uno está ahí también por interés propio (...) ahí uno como dirigente carga con muchos prejuicios como el de ser comumista, que tiene intereses propios, pero el de ser comista que tiene intereses propios, pero tambien carga con la responsabildad de que a uno le delegan muchas funciones, le delegan muchos roles (...)". (Hombre 1).

A partir de los elementos que señalan las y los jóvenes, es posible apreciar que la imagen que visualizan y/o la que identifican que proyectan, se vincula con las posibilidades de participación que observan en sus espacios de organización social, consruyendo posibilidades de fomentar su apuesta activa, en pos de fortalecer aquellas imágenes que desean desarrollar, y a la vez, finalizar con aquellos imaginarios que se relacionan con las creencias culturales en torno al rol que han desempeñado. Así, las imagenes que construyen se convierten en movilizadores de su accionar activo

b) Aspectos asociados a la participación y práctica política de los dirigentes estudiantiles en el Centro de Estudiantes

El desarrollo del tópico asociado a la participación y práctica política de los dirigentes estudiantiles universitarios se segmentó en cuatro dimensiones: propósito de la participación y práctica política; organización; acciones; y nociones - vivencias de participación política.

Los propósitos de la participación y práctica políti- ca son variados. Destaca la idea de instalar procesos de representatividad en el contexto universitario privado $^{7}$, ya que se posiciona como un espacio desafiante al considerar las construcciones previas (con sus respectivas cargas valóricas) en torno a él. Vinculado a lo anterior, la propia participación de los dirigentes busca el desarrollo de la participación de sus compañeros. Ellos se conciben como un eje de movilización.

En cuanto a instalar procesos de representatividad en la universidad, se dice que: "(...) estamos resguardando, (...) generar participación, generar puntos de encuentro entre los estudiantes, antiguamente nadie se conocía, yo ahora estoy en tercero y conozco a dos personas de quinto y ahora no pasa lo mismo que la gente de primero que conoce a todos los de segundo, muchos de tercero, de cuarto y así, generar participación, encuentro, y lo otro es que se instauren procesos ya de representatividad (...)". (Hombre 2).

Vinculado a los ejes expuestos, se hace mención a un propósito más amplio que apunta a ser garantes de derechos y de condiciones mínimas de calidad en sus espacios formativos universitarios. Resaltan la representatividad que poseen en sus roles, lo que posibilita un trabajo en pos de un aumento de la calidad de vida estudiantil de sus compañeros de carrera.

Asociado a ser garantes de calidad para sus compañeros representados, se agrega: "(...) yo creo que a nosotros nos movió el tema de calidad, de exigir calidad del servicio (...) hay mucha gente, sobre todo en la privadas que son los primeros en llegar a la universidad en su familia, por lo tanto uno se genera expectativas... uno tiene una carga, de partida, y eso también genera expectativas de lo que es la universidad. (..) cuando uno llega a una carrera donde no hay proces cuando uno theg académicas administrativas, económicas (...) ahí nos movió a hacer algo (...)". (Hombre 1)

La conjugación de la preocupación por romper con imaginarios estáticos en torno a la participación política en los escenarios construidos en las universidades priva defensa de los derechos de los estudiantes, posibilita visibilizar una configuración particular en torno a los alcances que la participación política de los dirigentes estudiantiles de Trabajo Social pueden desarrollar, pues en estas declaraciones conjugan elementos disciplinares.

Cobra particular relevancia, pues lo vinculan a las miradas tradicionales que las sociedades poseen en torno a estos espacios. Ya que se les conciben como "no participativos", pues la participacion politica estaria en los espacios brindados por las universidades públicas. He aqui la posibilidad de romper con una mirada estigmatizadora en torno a las posibilidades que poseen.
En relación a la organización interna, fundamentalmente desde la experiencia de los Centros de Estudiantes (CCEE), se relevaron tres aspectos: una alta valoración a una organización horizontal; el sistema de coordinadores que resultaba altamente funcional para uno de los CCEE, y el rol que cumple la asamblea dentro de su orgánica. Expresando el valor asignado a la horizontalidad en su organización, se plantea: "(...) nosotros no creemos en los cargos como jerarquizados, sino más bien en una organización más horizontal (...)”. (Hombre 3).

Se agrega: "(...) son cargos que están por el protocolo, pero nuestra idea es que nosotros cumplamos casi e mismo rol..., participemos de la misma forma, porque no creemos en la jerarquía y nuestro centro de alumno se va a caracterizar por eso (...)". (Mujer 1).

Respecto a las acciones o actividades concretas que los dirigentes estudiantiles universitarios realizan desde su propia participación y práctica política ellos destacan en relación a las características de sus acciones que estas sean concretas, realistas, no utóde sus compañeros y en la motivación a procesos de participación más amplios al interior de la carrera. Así lo ilustran: "(...) ahora estamos conformando los estatutos como para poder dejar algo para el futuro. para el siguiente centro de estudiantes...entre nuestro objetivos principales está la participación estudiantil, porque también es súper baja en la escuela y las actividades que hacemos siempre propenden a integrar motivar a los chiquillos (...)". (Hombre 1)

En la noción de participación política planteada por los jóvenes, esta es fuertemente diferenciada de la política partidista, enfatizando que más bien se vincula a lo que significa vivir en sociedad, a la mirada crítica de la misma y la expresión de la democracia. cipación política desde el CcEE, se explica que se rabaja en pro de velar por la garantía de ciertos derechos, crear espacios de representatividad dentro de la universidad, desde una lógica de integrar incluir a sus compañeros. Por otra parte, también se señala como parte de sus vivencias la baja participación de sus compañeros, en contraposición a la perseverancia del grupo del CcEE que es estable sistemático en participar

c) Opiniones respecto a los voceros y asambleas desde los dirigentes estudiantiles universitarios.

Respecto a las opiniones que los dirigentes estudianpicas. Por otra parte, están centrados en la inclusión En relación a la forma en que vivencian la parti- 
tiles universitarios emitieron respecto al sistema de voceros y asambleas, se desprendieron cuatro dimensiones: la valoracion al sistema; los facilitadores que ofrece; los obstaculizadores y la presencia de sistemas alternativos de organización.

En cuanto a la valoración, el sistema de voceros asamblea se valora positivamente, sin embargo se plantea como necesario el análisis del contexto en que se aplicará; la carrera, número estudiantes, jornadas, entre otros. También se hace alusión a la noción de asamblea, si se está hablando de una instancia multitudinaria o más acotada de diálogo grupal.

Por ejemplo, para uno de los CCEE no era totalmente pertinente el uso de la asamblea como mecanismo principal de participación y representación. Así lo explican: "(...) la realidad de nosotros versus la de ustedes, yo creo que pa' nosotros es mucho más difícil aunar criterios en una asamblea con intereses de vespertinos, que es un perfil de personas, a un perfil de esturtiantes de diuno une es otro tipo de per perfil de estuderes de diurno que es otrotipo de personas, entonces el sistema que nosotros llevamos (... [que] elegimos como mejor obviamente, por algo lo es tamos implementando (...) creo que el sistema de tener representantes, coordinadores por cada curso, es súper importante como se instaura el proceso para elegir a esos representantes y coordinadores (...)". (Hombre 2). Respecto a los facilitadores del sistema de asamblea, se destaca el poder de representación que puede alcanzar y la utilidad para la toma de decisiones: "(...) cada persona tiene una opinión, y que, si esa opinión es válida, tiene que decírsela al grupo, porque al fin y a cabo, fortalecer, tener una asamblea es para que todos puedan dar su opinión y que las decisiones sean tomadas por la máxima gente que vaya, la que quiere opina la que quiere tomar decisiones, (...) esa es nuestra línea y creemos que es la mejor (...)". (Hombre 4).

Se complementa con: "(...) lo que queremos es und cosa totalmente distinta que es la asamblea, en la asam blea pueden participar todas las personas que quieran entonces si después alguna persona me dice, oye pero por qué decidieron esto, estuvo en el correo, en todas partes que iba a haber una asamblea y que se ibma pacer estas decisiones, entoloce darles la responsabilidad a ellos, es tu responsabilida participar en la asamblea, la asamblea se va a hace los días que no haya clase entonces sí van a poder, y si hacemos asamblea en horario de clase vamos a intentar que se corten las clases, entonces va siendo una responsabilidad de los estudiantes participar en las decisiones (...)". (Hombre 3)
En relación a los obstaculizadores que puede tener el sistema de asamblea, se plantea que en ocasiones puede no ser representativa, si la participación en ellas es baja. Se señala: "(...) si bien esto es descentralizado, porque a la asamblea ojalá viniese harta gente y yo dudo que ahora vaya a venir tanta gente como cuando habian problemas graves y aparte que es fin de semestre (...) pueden haber diez personas, yo no podría tomar una decisión con diez personas porque la carrera es de quinientos (...) hay que complementar dos cosas nuevamente [sistema de coordinadores y asamblea] pero el tema de las asambleas no necesariamente me dice a mi que es democrático, porque por lo general en la asambleas se toman decisiones, no siempre estando (a) estando la mayoría y eso pa' mi va en contra de la democracia y tener coordinadores para mi sí es más representativo (...)". (Hombre 1)

De esta forma, uno de los CCEE plantea que el sistema de coordinadores por cursos es válido $\mathrm{y}$ complementario con la asamblea, mediando de alguna manera la noción de "asamblea" que se tenga. Así, van configurando formas de trabajo que se presentan como complementarias y/o diferentes, al sistema de representación tradicional ${ }^{8}$.

d) Opinión respecto a los partidos políticos y a los políticos desde la perspectiva de los dirigentes estudiantiles universitarios

Respecto a la opinión que los dirigentes universitarios tienen respecto a los partidos políticos y a los políticos, emergieron tres dimensiones: la disposición hacia ellos; la visión particular respecto a ellos, y los procesos que median la visión actual que poseen

En relación a la disposición hacia los partidos políticos y los políticos, señalan que votan, sin embargo lo hace por criterios racionales, analizando las propuestas de los políticos; no en base al partido o a la emocionalidad que eso les podría generar

En cuanto a la visión específica hacia los partidos políticos y los políticos, lo que apareció con más fuerza en los discursos apela a crisis de representatividad de ellos y a un evidente desgaste.

Lo grafican diciendo: "(...) Pa' mi hoy dia los partidos políticos están pasando por una crisis de representatividad, siento que mucha gente como dices tú no vota por los partidos, por lo mismo no se representan por ellos, entonces se dejan llevar por la persona, el fenómeno Bachelet, Bachelet por su simpatía, por su liderazgo carismático, digamos, está donde está y porque es mujer y porque es mujer separada, se identifica con las personas
(...) los partidos no están cumpliendo su rol, para mi se están beneficiando, adquiriendo beneficios personales (...)". (Mujer 2).

En relación a los procesos sociales que ellos consideran, median sus opiniones al respecto, destacan que la democracia no es representativa en Chile y que sus opiniones son parte de una opinión públi$\mathrm{ca}$, fuertemente afectada por lo que muestran los medios de comunicación.

Así lo explican: "Yo creo que ahí también estamos siendo súper cerrados en una mirada, porque los partidos do super cerrados no son solamente los que están en el congreso, no son solamente los que tienen una representación política como un diputado, un senador, como presidente, (...) la visión que nosotros tenemos de los partidos politicos que están como en la opinión pública todos los días (...) actualmente como decías tú, se están mezclando intereses, ya no estamos claros, por ejemplo, cual es la diferencia entre RN y la UDI, por ejemplo, cual es la diferencia entre a DC y UDS, por ejemplo, cual es la dferencia entre el DC y elPS, por ejemplo, siendo que antiguamente estaba marcado y por cosas del sistema, del sistema binominal, sistema democrático, la idiosincrasia chilena que fue después de los noventa, post-noventa se ha ido también encasillando". (Hombre 2).

e) Relación entre el Centro de Estudiantes y las Federaciones de Estudiantes.

Al indagar en la relación que los estudiantes y los CCEE a los que pertenecen poseen con sus respectivas Federaciones de Estudiantes (FFEE), emergieron cuatro dimensiones para el análisis: el tipo de Federación que poseían; su opinión específica respecto a la Federación; actividades que los asociaban a

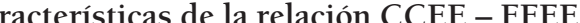
En cuanto al tipo de Federación, uno de los CCEE no contaba con la figura en su universidad, dado que su conformación exigía previamente una determinada cantidad de CCEE de las distintas carreras adscritas para ella; en este caso se operaba con un Consejo de CCEE. El otro CCEE sí contaba con la figura de FFEE

Lo explican: "Bueno, en la universidad [universidad $\left.N^{\circ} 2\right]$ no hay federación, no existe federación por un tema de estatutos que se necesitan más centros de alumnos de los que existen para conformar una federación, pero sí hay algo como federación, en lo poco que nosotros hemos participado, que has sido un mes... que esta (..) de juntarse de hacer cosas, cosa que el año pasado hacian muy pocas personas y que no se juntaban periódicamente (...)". (Hombre 3).

Para ambos CCEE la opinión relativa a la FfEE fue negativa, señalaron que sus preocupaciones son banales y que piensan al "estudiante" desde sus necesidades básicas.

Se señala: "(...) que es una constante al parecer en las privadas el tema de la banalidad, por decirlo de alguna manera, ... yo creo que pasa con la federaciones, (... ellos tiene un rol activo en cuanto a necesidades más básicas de un joven que de un universitario, tienen un concepción distinta de lo que es ser universitario que del centro de estudiantes y eso obviamente genera diferencias y diferencias grandes, nosotros con la federación no tenemos relación hasta ahora (...)". (Hombre 1).

Las actividades que se hacen en conjunto con los CCEE no fueron detalladas, sin bien se dice que "hacen cosas". Se tratan temas globales relativos a la vida universitaria. Destaca en ambos casos, una desconfianza a las unidades de Dirección de Asuntos Estudiantiles, desde donde -se explica- las FFEE son manejadas y controladas.

Al intentar caracterizar la relación entre los CCEE y las FFEE o sus símiles, se reconocen más diferencias que acuerdos, sin embargo los CCEE señalan mantener una relación estratégica con ellos, dadas las racilidades y los recursos disponibles para realizar ciertas acciones con los estudiantes de sus carreras de origen.

\section{Conclusiones y cuestionamientos}

Una vez presentados los ejes centrales del análisis construido, resulta interesante establecer alguna vinculaciones entre los hallazgos - entendiendo que se basa en un ejercicio de investigación acotado os referentes del fenómeno social desarrollados a inicio del texto.

Para efectos de este ejercicio de síntesis, se agruparán los tópicos en dos grandes ámbitos de reflexión el dirigente estudiantil universitario y la práctica política de éste.

En relación al dirigente estudiantil universitario el habitus de dirigente es constitutivo de identidad, una forma de verse a sí mismos y una forma en que son vistos por otros, apela a ámbitos de diferenciación al interior del campo. Lo que el dirigente es, desde su autodefinición apunta a lo que socialmente se espera de él: que represente, que medie, que lidere, etc. Por su parte, la forma en que lo ven da cuenta de las distintas posiciones de los agentes en el campo y de los diferentes ámbitos de diferenciación: respecto a sus compañeros, a la Federación de Estudiantes, a la universidad, al contexto extrauniversitario, entre otros. Ahora, es importante destacar que esta forma en que es concebido el rol 
del dirigente permite visibilizarlo como parte del movimiento de las resignificaciones que se le brindan en estos espacios, pues, como se menciono, traen consigo el peso de miradas fijas y prejuiciosas. En cuanto a la práctica política de los dirigentes estudiantiles universitarios, es importante señalar que ésta se encuentra situada en una estructura social, que va permeando los espacios cotidianos y locales. En el plano de lo político, impacta el cómo se está viviendo la política partidista hoy, el rol que cumplen los partidos políticos y los personeros políticos, y cómo este a fecta a las prácticas políticas Los jóvenes se desmarcan de los partidos políticos, diferencian su accionar, lo independizan; sin nega que estos están presentes, e incluso hacen mención a aquellos que no son relevados desde los medios de comunicación; pero son enfáticos en señalar que sus propósitos y acciones tienen referentes propios. En pencri, $y$ acciones tienen referentes propios. En son críticos con los partidos políticos apelando a crisis de representatividad y el desgaste de los mismos. Lo que es consistente con la indiferencia a los partidos políticos enunciada por la Quinta Encuesta Nacional de la Juventud (INJUV, 2008), donde cas un 37\% de los jóvenes no adscribe a ninguna corriente política.

Respecto a las nociones de participación política que acuñan y que les hace sentido, éstas también se desmarcan de la noción partidista. Lo plantean en un sentido amplio, como lo propio de vivir en sociedad, en democracia, se relaciona con la ciudadanía con el pensamiento crítico y con una política "informal", en sus palabras, que es altamente valorada, lo que se relaciona con los postulados de Saintout (2009), cuando señala que los jóvenes reconocen como "lo político" aquello asociado al encuentro con otros, de manera igualitaria y democrática, generando estructuras incluyentes. Esto también se vincula con la idea de "ciudadanía activa" (Gamboa \& Pincheira, 2009), donde la acción política se da en los espacios locales, en las situaciones cotidianas que identifican la subjetividad juvenil, y brinda posibilidad de comprender formas innovadoras de desarrollar procesos de responsabilidad, acción, y construcción democrática de los espacios en que se desarrolla la participación política de los dirigentes estudiantiles. Lo anterior permite reflexionar en torno a estas nuevas manifestaciones en tensión con los espacios que la propia carrera proporciona a las y los estudiantes, pues, como se ha mencionado, disciplina del Trabajo Social posee una constitución en lo político que destaca a lo largo de su desarrollo.
La práctica política de los dirigentes estudiantiles se desarrolla en un contexto específico, que es el universitario privado. En palabras de Bourdieu, ese es el campo en que se establecen los juegos y relaciones de las posiciones que los distintos agentes van desplegando, con sus van desplegando, con sus determinadas disposiciones. Este campo, a partir del ejercicio investigativo realizado, abre la posibilidad de revisarlo con detenimiento, pues configura en sí mismo la posibilidad de reconstruir las prácticas de lo político en su interior. No es posible olvidar que los orígenes de este espacio y su desarrollo configuran una serie de elementos que reflejan la sociedad que brinda ese contexto de participacion estereotipatan qux vez, provoca una posibilidad de movimiento particular a su interior, en donde las y los estudiantes desarrollan, desde sus significados, procesos particulares.

En relación a su práctica política propiamente tal, expresada en el trabajo que desarrollan en sus CCEE, gran parte de sus discursos se centrar en la parte organizativa de ella, entendiendo que esta forma concretiza una manera de pensar la participación desde los cánones más profundos que ellos
identifican.

Los propósitos de su práctica política se centran en generar participación entre sus compañeros, integrar, articular, instalar procesos de representatividad en el espacio universitario. Estos apuntan a lo que Gamboa \& Pincheira (2009), llaman la definición y los lugares de las politicidad, es decir, aquello que la agrupación busca alcanzar. Lo que se condice con los planteamientos de la CEPAL (2007) cuando apela a la valorización de la participación de las y los jóvenes y al trabajar a favor de ella. Esto sin descuidar la mirada atenta a resguardar los derechos de los estudiantes, especialmente aquellos que apuntan a exigir una educación de calidad. Este es uno de los puntos que marca fuertes diferencias con generaciones de dirigentes estudiantiles de décadas anteriores, donde el movimiento estudiantil era instrumento de emancipación (Benedicto, 2005). Hoy el moviendo en sí mismo potencia la autorrealización, la expresión de la subjetividad juvenil y los esfuerzos por generar participación. Ahora los jóvenes son los principalmente transformados (Saintout, 2009).

Otro elemento de la práctica política de los estudiantes fue la forma de organización que ellos explicitaron, en la que se valora la horizontalidad, donde cada quien es responsable de sí mismo (Saintout, 2009, Gamboa \& Pincheira, 2009). En relación a los dos CCEE que participaron, uno de ellos -dadas las características contextuales- se organizaban a través de coordinadores por curso, junto a estancias de Asambleas ampliadas dadas de manera esporádica en el tiempo. Mientras, el otro CEE hacía su apuesta exclusivamente por el sistema ión y representación por excelencia. Estas formas nos permiten apreciar la necesidad de observar y comprender las formas de organización y los sentidos que se imponen en ellos. Optar por una forma particular de organización política es reflejo de las configuraciones que se otorgan a estas posibilidades

En torno al debate de la validez de las "Asambleas", tomando el ejemplo del movimientos estudiantil secundario (Baeza, 2008) donde resultaron altamente efectivas, éstas se valoraban desde la fuerza de representación que tenían y lo útiles que resultaban para la toma de decisiones, haciendo hincapié en que su aplicación debía adecuarse a las condiciones contextuales en que se usaría.

En relación a las actividades que se pudiesen reconocer como propias de su práctica política, no se detallaro; más bien se relevó el que eran acciones reales, en pro de la participación y dirigidas a todos sus compañeros. Estas acciones responden a un tiempo, que es limitado (Gamboa \& Pincheira, 2009), la duración del mandato el CEE, por lo tanto las actividades deben realizarse en ese período, de tal forma de responder responsablemente a sus compañeros

Al analizar globalmente lo que emergió respecto a la práctica política de los jóvenes estudiantes, se pueden reconocer múltiples características de las culturas juveniles (Reguillo, 2000), en tanto instancias de diferenciación, de construcción de identidad, de cabida a la diversidad de estilos juveniles en un contexto altamente dinámico.

Al interior de las universidades, las federaciones de estudiantes son por naturaleza la instancia mayor de representación estudiantil. En este punto surgieron elementos que llamaron la atención Primero, en residades no había federación, sino un consejo que suplía sus funciones. Segundo, se señalaban relaciones tensionantes o cuestionadoras respecto al trabajo de la federación; planteándose que a través de las direcciones de asuntos estudiantiles estas eran manejadas conforme a tener control de los estudiantes. Este dato resulta novedoso, ya que según la Quinta Encuesta Nacional de la Juventud (2008), se señala que las universidades son un con un $62 \%$ de confianza.
De todas formas, la relación con las FFEE es proyectada desde lo estratégico que es mantener el vínculo por los recursos y oportunidades que eventualmente surgen a favor de sus compañeros.

Así, a partir de este acotado ejercicio de investigación surgen múltiples interrogantes a continuar desarrollando, ¿Qué características asumen otros CCEE de otras carreras? ¿Se visualizan diferencias entre CCEE de universidades privadas versus los de universidad públicas? ¿Cómo se proyectará el habitus del dirigente más allá de los límites de la universidad? ¿Qué dimensión de la subjetividad juvenil se desarrolla desde la práctica política? ¿Cómo impacta esto en el escenario social donde se ubican los jóvenes hoy?

Por otra parte, se aportan luces importantes a quienes se desempañan en ámbitos de acompañamiento a los estudiantes universitarios, ofreciendo sus subjetividades en el ámbito de la participación política, evidenciando recursos y capacidades que pueden er aprovechados para sus propios procesos de desarrollo personal y profesional.

\section{Bibliografía}

BAEZA, J. (2006). Demandas y organización de los estudiantes secundarios: una lectura sociologica mas all de fronteras y análisis coyunturales. En: Revista Temas Sociológicos, №11, pág. $263-298$.

BENEDICTO, J. (2005). El protagonismo cívico de los jóvenes: autonomía, participación y ciudadanía. Ponencia presentada al III Congreso de Ocio Juvenil, Dinamia.

BENEDICTO, J. y MORÁN, M. (2002). La construcción de una ciudadanía activa entre los jóvenes. Madrid NJUVE.

BOURDIEU, P. (1988). La distinción. Criterios y bases sociales del gusto. Madrid: Taurus.

EPAL. (2007). La juventud en Iberoamerica: tendencia y urgencias. Buenos Aires: CEPAL

CHAUVIRÉ, C. \& FONTAINE, O. (2008). El vocabulafio de Bourdieu. Buenos Aires: Atuel.

GAMBOA, A. \& PINCHEIRA, I. (2009). Organizaciones juveniles en Santiago de Chile. Invisibles Subterráneas. antiago: LOM

INJUV. (2008). Quinta Encuesta Nacional de la Juventud. Santiago: Gobierno de Chile.

MATURANA, S. (2007). Reflexiones en torno a la relación entre los jóvenes y la política a la luz del movimiento estudiantil secundario. En: Revista Central de Sociología, año 2, №2 
MARTÍNEZ, L., SILVA, C., MORANDÉ, M. y CANALES, L. (2010). Los jóvenes ciudadanos: reflexiones para Decala, No32, pag $105-118$. Década, №32, pág. $105-118$

MORÁN, M. Y BENEDICTO, J. (2000). Jóvenes y ciudadanos. Madrid: INJUVE.

RIZO, M. (2006). Conceptos para pensar lo urbano: el abordaje de la ciudad desde la identidad, el habitus y

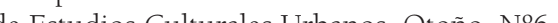

SAINTOUT, F. (2009). Jóvenes: el futuro llegó hace rato. Percepciones de un tiempo de cambios: familia, escuea, trabajo y politica. Buenos Aires: Prometeo.

\section{Políticas de reconstrucción posterremoto en Chile: dispositivo biopolítico, objetivación/subjetivación y resistencia'. Aproximaciones desde una lógica foucaultiana}

Reconstruction policies post-earthquake in Chile: bio-political device, objectivation / subjectivation and resistance.

Approximations from foucaultianan logic

Christian Arriagada D.

Licenciado en Trabajo Social. Magister @ en Trabajo Social. Correo electrónico: cnarriag@uc.

Resumen

El siguiente articulo presenta algunas aproximaciones a la política de reconstrucción desarrollada en Chile luego del terremoto/tsunami que afectó al pais el 27 de febrero del año 2010. Se propone la idea de dispositivo biopolítico desde una arista del pensamiento de Foucault como posibilidad interpretativa frente a las acciones politicas desarrolladas tras la catástrofe. Se intenta vislumbrar algunas de las lineas del dispositivo puesto en marcha, considerando los procesos de objetivación y subjetivación puestos en marcha por la politica, y, a su vez, las resistencias presentadas frente a dichos procesos.

Palabras clave. Reconstrucción, desastres, dispositivo, biopolitica.

\section{Abstract}

The following article presents some approximations to the politics of reconstruction developed in Chile after the earthquake / tsunami that struck the country on February 27, 2010. This paper proposes the idea of bio-political device, from an edge of Foucault's thought, as interpretative possibility to the political actions developed after the catastrophe. It is intend to show some lines of the device put in pace considering the processes of objectivation and subiectivation implemented by the policy and the resistance presented against these processes.

Key words. Reconstruction, disasters, device, bio-politics,

\section{Introducción}

El terremoto y tsunami sufrido en el país el 27 de tebrero de 2010, trajo numerosas consecuencias de destrucción en varias regiones y en diversas localidades tanto urbanas como rurales, especialmente en las regiones VII y VIII, que resultaron ser las más afectadas, sobre todo su lado costero (CEPAL, 2010, pág. 38)
El Gobierno de Chile puso en marcha un plan de reconstrucción para poder enfrentar la emergencia con la intención de paliar los diversos efectos que tuvo el megasismo en las zonas de mayor desastre (MINVU, 2011). Estas estrategias de reconstrucción del gobierno fueron acompañadas por otra serie de intervenciones del ámbito privado y de la sociedad civil ${ }^{2}$. De esta manera, 